\title{
BIOPSIA PROSTÁTICA TRANSRECTAL ECODIRIGIDA Y DOLOR. ESTUDIO PROSPECTIVO ALEATORIZADO COMPARANDO GEL LUBRICANTE, GEL CON LIDOCAÍNA Y BLOQUEO ANESTÉSICO DE HACES NEUROVASCULARES CON LIDOCAÍNA $1 \%$.
}

Enrique Argüelles Salido, Carmen B. Congregado Ruiz, José M. Conde Sánchez, Verónica Ruiz Zafra', Rafael A. Medina López y Pedro Campoy Martínez.

Servicio de Urología y Servicio de Anestesiología y Reanimación?. Hospitales Universitarios Virgen del Rocío. Sevilla. España.

Resumen.- OBJETIVO: Cuantificar ansiedad y dolor inmediato y tardio producidos, efectividad de los métodos analgésicos comunes y complicaciones.

MÉTODOS: 117 pacientes fueron distribuidos aleatoriamente en tres grupos: 1 (39): lubricante gel, 2 (38): lidocaína en gel intrarrectal, 3 (40): bloqueo anestésico con lidocaína al 1\%. Se recogieron las complicaciones y el dolor inmediatos y tardíos mediante una escala visual analógica.

RESULTADOS: $70 \%$ de pacientes estaban poco ansiosos. No encontramos diferencias significativas entre la analgesia conseguida en el grupo $1(4.37 \pm 2.41)$ y

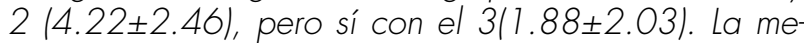
dia de dolor la tarde tras la prueba fue menor en el
Enrique Argüelles Salido Cardenal Bueno Monreal, 33 portal 2B - 6E 41013 Sevilla. (España). earguelles@telefonica.net Trabajo recibido: 3 de diciembre 2007 grupo $3(2.08 \pm 2.28)$ que en 1 y $2(4.06 \pm 2.80$ y $2.42 \pm 2.031$, pero sin significación estadística. Los pacientes no mostraron un dolor distinto al paso del transductor transrectal, oscilando entre 2.34 para el grupo 3 y 3.38 para el grupo 2. Agrupados según tono estinteriano, si encontramos diferencias (bajo 2.71 2.76 ,

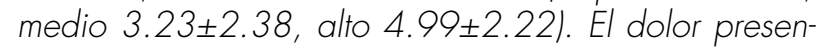
tado por los pacientes que decían estar muy nerviosos pre-prueba fue de 4.98 2.57, significativamente mayor que el presentado por los que decían estar nada nerviosos $(2.59 \pm 2.21)$. No encontramos diferencias en las complicaciones entre los tres grupos.

CONCLUSIONES: El empleo de gel intrarrectal de lidocaína no ha demostrado en nuestra serie ser más eficaz que el uso de lubricante para el control del dolor, pero si la lidocaína inyectada en ápex y vesículas seminales. Tono esfinteriano y ansiedad preprueba son los factores más determinantes en el grado de dolor.

Palabras clave: Próstata. Dolor. Biopsia. Transrectal. Lidocaína. Analgesia. Anestesia.

Summary.- OBJECTIVES: To quantify anxiety and immediate and late pain, efficacy of the common analgesic methods, and complications.

METHODS: 117 patients were randomized to three groups: 1(39): lubricant gel; 2 (38): intrarectal lidocaine gel; 3 (40): anesthetic blockage with 1\% lidocaine. Complications and immediate and late pain were collected, these latter with a visual analogic scale.

RESULTS: $70 \%$ of the patients were not much anxious. We did not find significant differences between the analgesia achieved in group 1 (4.37 2.41 ) and group 


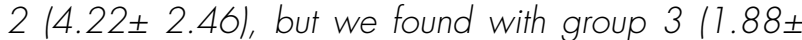
2.03). Mean pain score the afternoon after biopsy was lower in group $3(2.08 \pm 2.28)$ than in groups 1 and 2 (4.06 \pm 2.80 and $2.42 \pm 2.03$ ), without statistical significance. The patients did not show a difference in pain during the pass of the transrectal transducer, varying between 2.34 for group 3 and 3.38 for group 2. Grouped by sphincter tone we find differences llow $2.71 \pm 2.76$, medium 3.23 2.38 , high 4.99+- 2.22). Patients feeling very nervous before the test had a pain score of $4.98 \pm 2.57$, significantly greater than the pain of those not feeling nervous $(2.59 \pm 2.211$. We did not find differences in complications between the three groups.

CONCLUSIONS: The use of intrarectal lidocaine gel did not demonstrated being more effective in pain control in our series than the use of lubricant gel, but lidocaine injected into the apex and seminal vesicles was. Sphincter tone and pre-test anxiety are the most determinants factors for pain.

Keywords: Prostate. Pain. Biopsy. Transrectal. Lidocaine. Analgesia. Anesthesia.

\section{INTRODUCCIÓN}

En España, durante los últimos años, el cáncer de próstata (CP) se ha situado en el tercer lugar como causa de muerte por neoplasia en varones, tras los tumores de pulmón y los colorrectales, aunque en 2004 presentó un incremento del 1\% que le hizo situarse puntualmente en segunda posición, desbancando a estos últimos. En 2005 provocó en España 5511 defunciones, un 3.2\% menos que en el año anterior (1). En nuestro país, la evolución de las tasas de mortalidad ha sido ascendente desde los años 50, si bien la tendencia se suavizó a partir de 1970. Globalmente, se observa un incremento medio en torno al $1 \%$ anual, con un cambio en la tendencia en el año 1998, a partir del cual la mortalidad empiezó a disminuir, con una tasa estandarizada por edad de 22,9 muertes por 100.000 habitantes en 2000 (2).

Aproximadamente dos tercios de los pacientes presentan el tumor confinado al órgano en el momento del diagnóstico, y son por tanto subsidiarios de tratamiento radical con fines curativos. El $20 \%$ son tumores localmente avanzados y el $5 \%$ restante son tumores diseminados (3).

Por todo ello en los últimos años el diagnóstico precoz de este tumor se ha convertido en un objetivo fundamental para el urólogo, y la ecografía transrectal con toma de biopsia (ECO-TRB) en el método de diagnóstico fundamental para ello.
Inicialmente se consideró este procedimiento como no doloroso debido a la "relativa insensibilidad de la pared rectal anterior", no empleándose anestesia alguna en su realización. Sin embargo, a medida que se avanzó en el diagnóstico precoz del $\mathrm{CP}$, la biopsia sextante clásica dio paso a modelos de biopsia ampliada. Ésto y la frecuente necesidad de repetir la toma de biopsias en más de una ocasión ha hecho surgir la necesidad de una analgesia efectiva y con un número de complicaciones limitado (4).

\section{Nos proponemos en nuestro trabajo:}

\section{- Objetivos principales:}

- Cuantificar el dolor y la ansiedad producida por este procedimiento diagnóstico, tanto durante la realización de la prueba como en las horas posteriores, valorando la posible relación entre ellos.

- Valorar la efectividad de los métodos analgésicos más comúnmente empleados.

- Cuantificar los efectos secundarios del procedimiento en nuestra serie, tanto de manera inmediata a la prueba como tardía, relacionándolos con el tipo de anestésico administrado.

\section{- Objetivos secundarios:}

- Analizar si existe relación entre las molestias experimentadas y edad o volumen prostático.

- Valorar cuánto del dolor experimentado se debe a la dilatación anal debida al transductor y cuánto a la toma de biopsia en sí.

\section{MATERIAL Y MÉTODOS}

\section{Criterios de selección}

La indicación de ECO-TRB se realizó conforme a las directrices establecidas por el Proceso Asistencial Integrado Hiperplasia Prostática BenignaCáncer de Próstata de la Consejería de Salud andaluza (5). Se indicó biopsia inicial en pacientes con:

- PSA entre 3-10 ngr/mL, con índice libre menor o igual a $20 \%$.

- PSA mayor de $10 \mathrm{ngr} / \mathrm{mL}$.

- tacto rectal positivo.

Se indicó rebiopsia en pacientes con:

- Tacto rectal positivo.

- PSA mayor de $10 \mathrm{ngr} / \mathrm{mL}$.

- Neoplasia intraepitelial de alto grado.

- Atipia.

- PSA velocidad >0,75 y/o >20\% PSA basal.

- PSA 3-10. L/T<0,15 
Como criterios de exclusión se establecieron:

- Cirugía rectal previa.

- Hemorroides grado mayor de dos (protusión espontánea no relacionada con esfuerzos).

- Fisura anal o en general procesos proctológicos agudos.

- Negativa del paciente a colaborar en el estudio.

- Alergia a anestésicos locales.

- Presencia de dolor agudo por algún motivo no proctológico, fundamentalmente lumbar provocado por la posición necesaria para la realización de la prueba.

\section{Pacientes y agrupamiento de la muestra}

Se crearon 3 grupos de pacientes en función de la analgesia administrada:

- grupo 1: lubricante gel (aquage|囚)

- grupo 2: lidocaína en gel intrarrectal (cathejell囚).

- grupo 3: bloqueo anestésico con lidocaína al 1\%, inyectando $5 \mathrm{cc}$ en ápex prostático y $2.5 \mathrm{cc}$. en cada ángulo vesículo-prostático.

Para demostrar una diferencia del $20 \%$ en la variable "dolor producido por la biopsia" con una desviación estándar de 2 puntos en la escala de 1 a 10 con una potencia del $85 \%$ y un error alfa del $1 \%$ se establece para una hipótesis de dos colas un mínimo de 27 pacientes por grupo, completados con una proporción de seguridad del $33 \%$ en cada grupo en previsión de posibles pérdidas.

De 125 pacientes consecutivos, 117 pacientes cumplieron los criterios de inclusión y concedieron su consentimiento informado, siendo distribuidos de manera aleatoria en los grupos 1 (39 pacientes), 2 (38 pacientes) y $3(40$ pacientes).

\section{Procedimiento de ECO-TRB}

Todos los pacientes recibieron, la noche antes de la intervención y la mañana del día de ésta, una profilaxis consistente en ciprofloxacino $500 \mathrm{mgr}$. vía oral y enema de dihidrógeno fosfato sódico (enema Cassen $($ ). Los procedimientos diagnósticos fueron realizados indistintamente por tres investigadores entrenados de manera homogénea antes de comenzar el estudio.

Los pacientes fueron colocados en posición de decúbito lateral izquierdo con cadera flexionada. Inicialmente se realizó un tacto rectal, usando como lubricante en todos los casos aquagel $(\AA$ y posteriormente se realizó la exploración ecográfica midiéndose el volumen y características prostáticas y periprostáticas, así como estado de conservación capsular y de ángulos seminoprostáticos.
En el grupo 1 tras la exploración digital se volvía a administrar aquagel $\circledast$, intrarrectal, previamente a la introducción de la sonda ecográfica. En el grupo 2 se empleaba en este momento gel de lidocaína, esperando cinco minutos hasta comenzar la toma de biopsia, tiempo en el que se realizaron las mediciones prostáticas oportunas. En el grupo 3 la inyección de lidocaína se realizó posteriormente a la realización de las mediciones prostáticas, porque éstas pueden verse afectadas por la compresión ejercida por el habón anestésico, fundamentalmente en la zona del ápex.

En los pacientes biopsiados por primera vez se tomaron 8 cilindros, mientras que en las rebiopsias se tomaron 10 cilindros. Para ello se empleó un transductor multiplanar de $7 \mathrm{MHz}$. y canal de punción integrado. Las muestras fueron tomadas mediante punción automática con aguja de $22 \mathrm{~mm}$. de longitud y $18 \mathrm{G}$ de grosor.

\section{Procedimiento de bloqueo de la inervación peripros- tática}

Con una aguja de $20 \mathrm{G}$ y $28 \mathrm{~cm}$., bajo visión ecográfica y empleando lidocaína al $1 \%$, se introdujeron 2.5 cc. en cada ángulo vesículo-prostático, en la zona triangular denominada por algunos autores "signo del Everest", y posteriormente 5 cc. en la zona central del ápex prostático, bajo la fascia de Denonvillers, con aspiración previa para detectar una posible inyección intravascular accidental.

\section{Recogida de datos}

Se realizó en dos pasos: durante la realización de la prueba el investigador rellenó la hoja de recogida de datos 1 en el que fundamentalmente se recogieron variables demográficas, y las que hacen referencia a la ansiedad y dolor provocados por la prueba, cuantificadas según la escala visual analógica del dolor horizontal (EVA), graduada de 0 a 10, acotada en sus extremos. También se recogieron en este momento las complicaciones inmediatas (Figura 1). Al finalizar la prueba se explicaba y entregaba un cuestionario en el que se recogía el dolor experimentado en la tarde de la biopsia, también con EVA, además de las complicaciones tardías del procedimiento (Figura 2), debiendo ser devuelto en la consulta posterior, cuando los pacientes acudieron a recibir los resultados anatomopatológicos.

Los datos obtenidos fueron recogidos y analizados mediante el programa R-Sigma, de Horus Hardware, emplendo el test de ANOVA para variables cuantitativas, Chi cuadrado para las cualitativas, y análisis de correlación para el estudio de relación entre variables cuantitativas. 


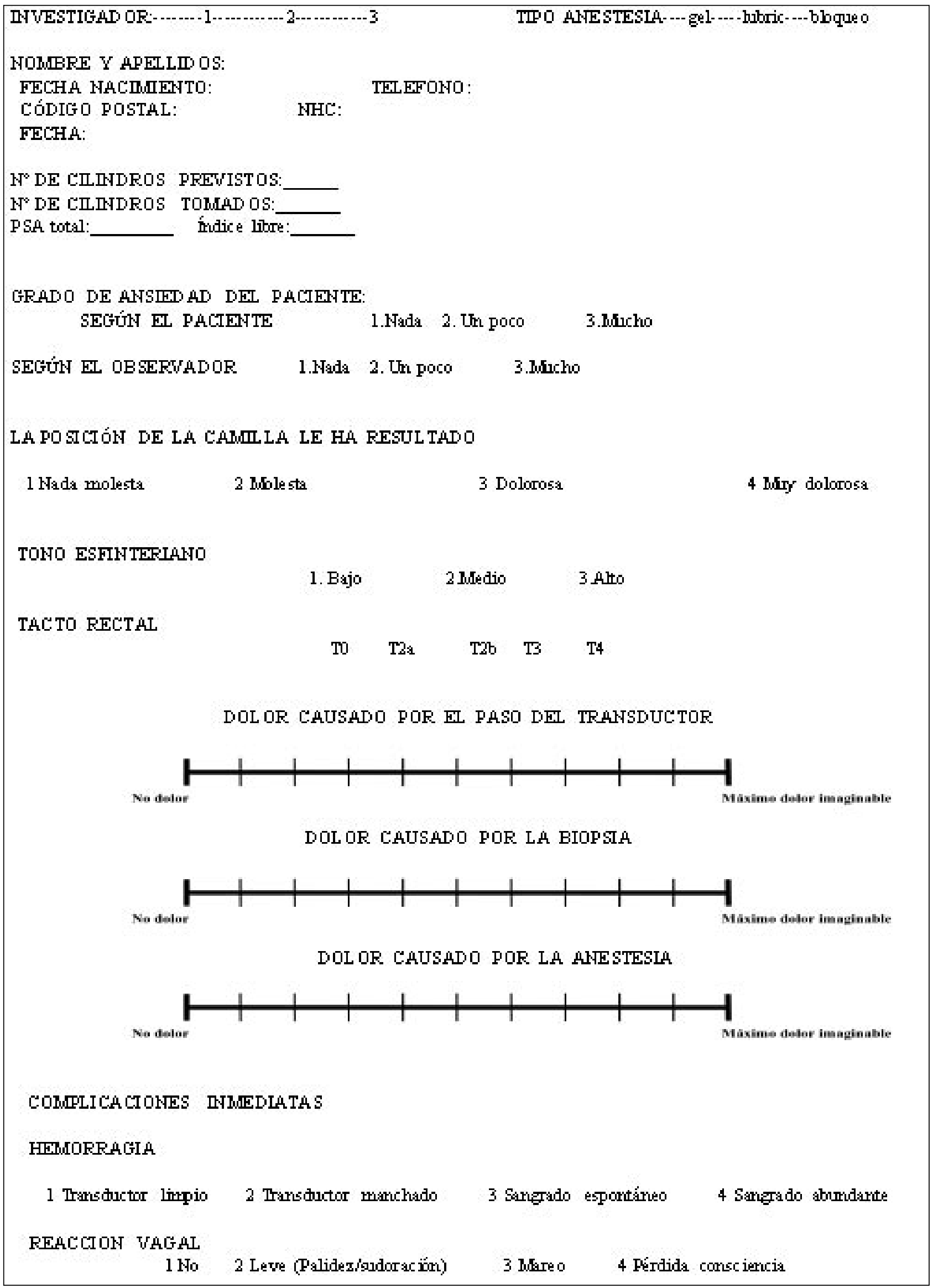




\section{MOÁRE Y AFELCLOS}

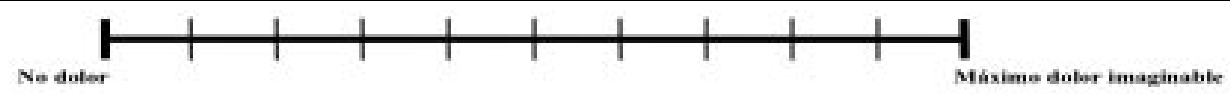

¿HA TOMADO ALGO PARA EL DOLOR DESPUÉS DE LA BIOPSIA? -------SI----------NO

SI HA MARCADO “Sí" ESCRIBA POR FAVOR QUÉ MEDICAMENTO HA TOMADO, Y CUANTOS DIAS

¿HA SANGRADO POR EL ANO? (marque lo que sea)

SINO

SI HA MARCADO "SÍ" APUNTE:

- DIA DE INICIO DEL SANGRADO POR ANO:

DIA DE DESAPARICION DEL SANGRADO POR ANO:

¿HA TENIDO FIEBRE?

SI-NO

SI HA MARCADO "SÍ" DÍGANOS

- CUAL HA SIDO LA TEMPERATURA MAYOR QUE TUVO?:

- DIA DE APARICIÓN DE LA FIEBRE:

- DIA DE DESAPARICIÓN DE LA FIEBRE:

¿QUÉ TOMÓ PARA LA FIEBRE?

¿HA SANGRADO AL ORINAR? ?

-SINO

SI HA MARCADO "SÍ" DÍGANOS

- DIA DE APARICIÓN DE LA SANGRE EN LA ORINA:

- DIA DE DESAPARICIÓN DE LA SANGRE EN LA ORINA:

¿HA VISTO SANGRE EN EL SEMEN?

1. Si 2. No 3. No he podido verlo/ no lo sé.

¿HA TENIDO DIFICULTADES PARA ORINAR?

1.No 2. Un poco3. Bastante 4. Mucho 5.Me han tenido que sondar

¿HA TENIDO ESCOZOR AL ORINAR?

1.No 2. Un poco 3. Bastante 4.Mucho

¿HA TENIDO QUE IR A URGENCIAS EN LOS DIAS SIGUIENTES A LA BIOPSIA?

1. $\mathrm{Si}$ 2. No

INDIQUENOS SUS COMENTARIOS 


\section{RESULTADOS}

La edad media de los 117 pacientes fue de 64.14 (desv. típica 6.35, rango 48-78). 70 de ellos $(59.82 \%)$ entregaron el cuestionario sobre dolor y complicaciones tardías.

Las características de estos grupos están resumidas en la Tabla I. No se encontraron diferencias estadísticamente significativas entre los grupos en cuanto a edad, PSA, tacto rectal, volumen prostático o tono esfinteriano.

\section{DOLOR Y ANSIEDAD}

En términos generales los pacientes no presentaban una excesiva ansiedad pre-prueba, superando en todos los grupos el $70 \%$ la suma de pacientes que se encontraban nada o poco ansiosos ante el procedimiento, tanto desde el punto de vista del paciente como del observador. Encontramos cierta diferencia respecto al porcentaje de pacientes que dicen no sentirse nerviosos ante la perspectiva de la prueba en el grupo 3 respecto a los grupos 1 y 2 , que atribuimos a la imposibilidad de hacer el estudio de manera ciega $135.8 \%$ en el grupo $1,21.6 \%$ en el grupo 2, 46.1\% en el 3, p>0.01). De esta forma tener conciencia de que se les estaba inyectando anestesia debe haber tenido un cierto efecto tranquilizador.

Comparando los distintos métodos analgésicos empleados y el dolor provocado por la toma de biopsias, no encontramos diferencias estadísticamente significativas entre el placebo (aquagel,) y el gel de lidocaína, con los que se producía un dolor medio de 4.37 y 4.22 respectivamente en una escala de 10. Sin embargo sí que había diferencias entre ellos y el bloqueo paraprostático con lidocaína, con una media de dolor de 1.88 sobre 10. Hay que destacar que el dolor medio producido por la inyección de la lidocaína se situó en $2.09 \pm 0.37$ (0-10), sensiblemente inferior al dolor producido por el procedimiento en los grupos 1 y 2 . Aunque la media de dolor la tarde tras la prueba también fue menor en los pacientes con bloqueo periprostático $(2.08 \pm 2.28)$ que en los grupos 1 y $2(4.06 \pm 2.80$ y $2.42 \pm 2.03$ respectivamente), no encontramos diferencias estadísticamente significativas (Tabla II). El $74.28 \%$ no precisó analgesia alguna durante dicha tarde.

TABLA I. CARACTERISTICAS DE LA MUESTRA.

\begin{tabular}{|c|c|c|c|c|}
\hline & Grupo 1 (39) & Grupo 2 (38) & Grupo 3 (40) & $\mathbf{p}$ \\
\hline $\begin{array}{c}\text { Edad } \\
\text { (media, rango) }\end{array}$ & $\begin{array}{c}66.4 \pm 6 \\
51-78\end{array}$ & $\begin{array}{c}65.6 \pm 5.7 \\
49-77\end{array}$ & $\begin{array}{c}63.9 \pm 7 \\
48-77\end{array}$ & n.s. \\
\hline PSA & $\begin{array}{l}10.82 \pm 7.41 \\
3.53-46.45\end{array}$ & $\begin{array}{c}11.25 \pm 15.08 \\
3.21-85.86\end{array}$ & $\begin{array}{c}11.22 \pm 20.05 \\
3.09-130\end{array}$ & n.s. \\
\hline Tacto rectal & $\begin{array}{c}\text { T0: } 81.5 \% \\
\text { T2a: } 13.1 \% \\
\text { T2b: } 2.7 \% \\
\text { T3: } 2.7 \%\end{array}$ & $\begin{array}{c}\text { T0: } 84.2 \% \\
\text { T2a: } 15.8 \% \\
\text { T2b: } 0 \%\end{array}$ & $\begin{array}{l}\text { TO: } 87.5 \% \\
\text { T2a: } 12.5 \%\end{array}$ & n.s. \\
\hline Tono esfínter & $\begin{array}{l}\text { Bajo: } 28.2 \% \\
\text { Medio: } 59 \% \\
\text { Alto: } \mathbf{1 2 . 8 \%}\end{array}$ & $\begin{array}{c}\text { Bajo: } 23.6 \% \\
\text { Medio:52.7\% } \\
\text { Alto: } 23.7 \%\end{array}$ & $\begin{array}{c}\text { Bajo: } 36 \% \\
\text { Medio: } 54 \% \\
\text { Alto: } 10 \%\end{array}$ & n.s. \\
\hline Volumen & $\begin{array}{c}45.68 \pm 3.21 \\
16.1-101\end{array}$ & $\begin{array}{c}50.63 \pm 5.13 \\
16.5-181\end{array}$ & $\begin{array}{c}54.02 \pm 3.84 \\
19.7-130\end{array}$ & n.s. \\
\hline
\end{tabular}

n.s.: no significativo 
Los pacientes no mostraron un dolor significativamente distinto al paso del transductor transrectal, oscilando entre 2.34 para el grupo 3 y 3.38 para el grupo 2. Sin embargo, si agrupamos el dolor causado por el transductor según el tono esfinteriano presentado, sí encontramos diferencias significativas. De esta manera en el grupo con tono esfinteriano bajo el valor medio encontrado fue $2.71 \pm 2.56(0$ $10)$, mientras que en los pacientes con tono alto fue de $4.99 \pm 2.22$ (0.4-8.4) (p.0.01). En el grupo de tono medio la media de dolor fue de $3.23 \pm 2.38$ (010), no mostrando diferencias significativas respecto a los otros dos grupos según la potencia definida. Sin embargo el tono esfinteriano no provocó diferencias significativas en el dolor provocado por la toma de muestras prostáticas (Tabla III).

Otros aspectos entre los que no encontramos una relación significativa fueron:

- Dolor provocado por la biopsia-edad: coeficiente de correlación (cc) 0.14, error estándar (ee) 0.09

- Dolor provocado por la biopsia-volumen prostático: cc 0.14 , ee 0.09

- Dolor provocado por el paso del transductor-edad: cc -0.01, ee 0.09

- Dolor provocado por el paso del transductor-volumen prostático cc 0.04, ee 0.09
Únicamente en dos pacientes (1.72\%), ambos del grupo 2, no fue posible la consecución del número de cilindros prefijados, debido al dolor provocado por la prueba.

Otro importante factor que determinó diferencias significativas en el dolor provocado por la toma de cilindros fue la ansiedad subjetiva preprueba. De esta manera el dolor presentado por los pacientes que decían estar muy nerviosos preprueba fue de $4.98 \pm 2.57$ (0-7), significativamente mayor que el presentado por los que decían estar nada nerviosos $(2.59 \pm 2.21$, rango $0-7)(p \cdot 0.01)$, y marcadamente superior aunque de manera no significativa con los que decían estar poco nerviosos $(3.51 \pm 2.53$, rango $0-10)(p>0,01)$. Sin embargo no encontramos diferencias entre el dolor provocado por el paso del transductor y el grado de ansiedad pre-prueba (Tabla IV).

\section{COMPLICACIONES}

No hemos encontramos diferencias entre los grupos, ni en las inmediatas ni en las tardías. Al retirar el transductor sólo el $31.9 \%$ de los pacientes no presentaba rectorragia en absoluto. El transductor estaba manchado de sangre en el $62.93 \%$ y en un

TABLA II. DOLOR EN DISTINTOS MOMENTOS.

\begin{tabular}{|c|c|c|c|c|}
\hline & Grupo 1 & Grupo 2 & Grupo 3 & p \\
\hline $\begin{array}{c}\text { Durante la toma } \\
\text { de biopsia }\end{array}$ & $\begin{array}{l}x=4.37 \\
\sigma=2.41 \\
R=0-10\end{array}$ & $\begin{array}{l}x=4.22 \\
\sigma=2.46 \\
R=0-8.5\end{array}$ & $\begin{array}{l}x=1.88 \\
\sigma=2.03 \\
R=0-7.5\end{array}$ & sign. \\
\hline $\begin{array}{l}\text { Durante el paso } \\
\text { del transductor }\end{array}$ & $\begin{array}{l}x=3.29 \\
\sigma=2.73 \\
R=0-10\end{array}$ & $\begin{array}{l}x=3.38 \\
\sigma=2.29 \\
R=0-8.2\end{array}$ & $\begin{array}{l}x=2.34 \\
\sigma=2.21 \\
R=0-10\end{array}$ & n.s. \\
\hline $\begin{array}{l}\text { Durate la } \\
\text { inyección } \\
\text { anestesica }\end{array}$ & 0 & 0 & $\begin{array}{l}x=2.09 \\
\sigma=0.37 \\
R=0-10\end{array}$ & n.s. \\
\hline $\begin{array}{l}\text { La tarde tras el } \\
\text { procedimiento }\end{array}$ & $\begin{array}{l}x=4.06 \\
\sigma=2.80 \\
R=0-10\end{array}$ & $\begin{array}{c}x=2.42 \\
\sigma=2.03 \\
R=0-5\end{array}$ & $\begin{array}{c}x=2.08 \\
\sigma=2.28 \\
R=0-6\end{array}$ & \\
\hline
\end{tabular}

$x=$ media $; \sigma=$ desv típica; $R=$ rango, sign=significativo, n.s.=no sign. 
TABLA III. DOLOR EN RELACIÓN A TONO ESFÍNTERIANO.

\begin{tabular}{|c|c|c|c|c|}
\hline & Bajo & Medio & Alto & p \\
\hline Dolor transductor/ & $\mathrm{x}=2.71$ & $\mathrm{x}=3.23$ & $\mathrm{x}=4.99$ & \\
tono esfínter & $\sigma=2.56$ & $\sigma=2.38$ & $\sigma=2.22$ & sign. \\
& $\mathrm{R}=0-10$ & $\mathrm{R}=0-10$ & $\mathrm{R}=0.4-8.4$ & \\
\hline Dolor biopsia/ & $\mathrm{X}=3.06$ & $\mathrm{x}=3.56$ & $\mathrm{x}=4.30$ & \\
tono esfínter & $\sigma=2.71$ & $\sigma=2.48$ & $\sigma=2.38$ & n.s. \\
& $\mathrm{R}=0-10$ & $\mathrm{R}=0-9$ & $\mathrm{R}=0-9.5$ & \\
\hline
\end{tabular}

$x=$ media $; \sigma=$ desv típica; $R=$ rango, sign=significativo, n.s.=no sign.

$5.18 \%$ había sangrado espontáneo, que en ningún caso fue abundante. Sólo el $5.16 \%$ de los pacientes tuvieron cierta reacción vagal, del tipo sequedad de boca, cierto mareo o sudoración, pero ninguno presentó pérdida de consciencia (Tabla V).

El 38.23\% de los pacientes presentó algún tipo de emisión de sangre por recto en casa. La duración media de este sangrado fue de $0.8 \pm 0.19$ días (desviación típica 1.53). El 61.19\% presentó hematuria, con una duración media de $2.37 \pm 0.44$ días (dt 3.61).

Sólo un paciente presentó fiebre tras la prue$\mathrm{ba}$, precisando tratamiento antipirético y antibiótico, con evolución satisfactoria. El $33.83 \%$ tuvo algún tipo de sensación miccional obstructiva, que fue leve en el $86.95 \%$ de ellos. Sólo un paciente precisó sondaje vesical. El $65.22 \%$ de los biopsiados refirieron no haber tenido disuria en las micciones posteriores a la prueba. El $31.88 \%$ la tuvo de tipo leve, y sólo dos pacientes tuvieron molestias más severas.

El $50.79 \%$ de los pacientes refiere hemospermia. De los pacientes restantes la mitad no tuvo ocasión de observarlo, y la otra mitad no la presentó (Tabla VI).

\section{DISCUSIÓN}

La biopsia prostática sextante mediante ecografía transrectal, tras su descripción por Hodge y cols. en 1989, se convirtió rápidamente en la técnica de elección para el diagnóstico de las neoplasias prostáticas. Desde entonces la técnica fue evolucionando, discutiéndose sobre sus indicaciones precisas y sobre el número de cilindros ideales a tomar. Sin embargo, el interés en el confort del paciente durante esta prueba no experimentó una evolución pareja.

TABLA N. DOLOR EN RELACIÓN A ANSIEDAD PRE-PRUEBA.

\begin{tabular}{|c|c|c|c|c|}
\hline & Nada & Poco & Mucho & P \\
\hline Dolor transductor/ & $\mathrm{x}=3.16$ & $\mathrm{x}=3.22$ & $\mathrm{x}=3.4$ & $\mathrm{n}$. .s. \\
ansiedad & $\sigma=2.81$ & $\sigma=2.41$ & $\sigma=2.02$ & $\mathrm{R}=0-7.5$ \\
& $\mathrm{R}=0-10$ & $\mathrm{R}=0-9$ & $\mathrm{x}=4.98$ & \\
\hline Dolor biopsia/ & $\mathrm{x}=2.59$ & $\mathrm{x}=3.51$ & $\sigma=2.57$ & sign. \\
ansiedad & $\sigma=2.21$ & $\sigma=2.53$ & $\mathrm{R}=0-9$ & \\
\hline
\end{tabular}

$x=$ media; $\sigma=$ desv típica; $R=$ rango, sign=significativo, $n . s .=$ no sign. 
Durante años se realizaba sin administrar analgesia alguna. En 1996 Nash y colaboradores, basándose en una técnica descrita por Reddy y cols. en 1990 para la dilatación uretral con balón, publican una técnica de bloqueo de la inervación prostática consistente en la inyección de lidocaína al $1 \%$ en el ángulo formado entre las vesículas seminales y la base prostática (6). Esta técnica no tuvo demasiado eco en la comunidad urológica hasta que en 2000 Soloway y cols. publicaron una serie de 50 pacientes que recibieron $5 \mathrm{ml}$ de lidocaína al $1 \%$ en el plexo neurovascular periprostático, obteniendo buenos resultados (7).

Se ha escrito mucho sobre la tolerabilidad de la prueba. La vía transrectal y sus connotaciones, además de la dilatación anal que el transductor provoca, el dolor despertado por la toma de biopsias, y la ansiedad que suscita la posibilidad de detección de una neoplasia maligna, hacen necesaria una analgesia eficaz y segura. La sensación dolorosa provocada por la prueba viene mediada por:

- La estimulación de la pared rectal, inervada por el nervio pudendo, originado en el plexo sacro, que provee de ramas a la porción anal del recto por debajo de la línea dentada, al esfínter estriado y a la zona cutánea perineal.

- La punción de la cápsula prostática. Su inervación proviene del nervio pélvico, procedente del plexo hipogástrico inferior. Contiene fibras autonómicas y sensitivas para la próstata y otros órganos adyacentes. Estudios en cadáveres han determinado que estas vias nerviosas se originan en el extremo de las vesículas seminales y discurren entre la próstata y el recto en el borde inferolateral de la próstata.

En nuestro trabajo hemos pretendido ahondar un poco en la importancia de cada uno de los componentes antes mencionados que influyen en la tolerancia a esta prueba. De esta manera destacamos que en los grupos 1 y 2 el factor más molesto de la prueba fue la propia toma de biopsias (media de 4.37 y 4.22 frente a 3.29 y 3.38 para el paso del transductor), mientras que en el grupo 3 , el empleo del bloqueo con lidocaína hizo que el paso del transductor transrectal se convirtiese en lo más doloroso (1.88 la biopsia/2.34 el transductor). Nos interesaba también cuantificar aisladamente el dolor provocado por la inyección del anestésico, pues de nada serviría que la toma de biopsia con lidocaína inyectada fuese escasamente dolorosa si la propia inyección de anestésico sí que lo fuese, tal como sugería el trabajo de Schostak y cols. (8). Al igual que en dicho trabajo en el nuestro la inyección de anestesia ha resultado más dolorosa que la toma de biopsia (2.09 frente a 1.88) pero menos que la toma de biopsia en cualquiera de los otros grupos. Öbek sugiere el empleo combinado de lidocaína perianal e intrarrectal con bloqueo periprostático para mejorar este aspecto (9).

En algunos estudios se hace referencia a la influencia de factores como la edad o el número de cilindros tomados en el dolor percibido. Djavan encontró un patrón de dependencia según la edad, de manera que los pacientes menores de sesenta años

TABLA V. COMPLICACIONES INMEDIATAS.

\begin{tabular}{|c|c|c|c|c|}
\hline & & GRUPO 1 & GRUPO 2 & GRUPO 3 \\
\hline \multirow{4}{*}{ RECTORRAGIA } & LIMPIO (31.9\%) & 9 & 12 & 16 \\
\cline { 2 - 5 } & MANCHADO (62.93\%) & 26 & 24 & 23 \\
\cline { 2 - 5 } & ESPONTÁNEO (5.17\%) & 4 & 1 & 1 \\
\cline { 2 - 5 } & ABUNDANTE (0\%) & 0 & 0 & 0 \\
\cline { 2 - 5 } REACCIÓN & NO (94.84\%) & 37 & 34 & 39 \\
\cline { 2 - 5 } & $\operatorname{LEVE}(2.58 \%)$ & 2 & 1 & 0 \\
\cline { 2 - 5 } & MAREO (2.58\%) & 0 & 2 & 1 \\
\cline { 2 - 5 } & COMA (0\%) & 0 & 0 & 0 \\
\hline
\end{tabular}


tenían una tasa de discomfort mayor que los mayores (10). También Rodríguez y Terris la encontraron (11). En nuestro trabajo no hemos visto que la edad sea un factor determinante. Tampoco Feltes y colaboradores, en un reciente artículo, la encuentran (12). Respecto al número de biopsias tomadas nos parece interesante el concepto de "dolor acumulado", introducido por Kaver y colaboradores, haciendo referencia a la sensación creciente de dolor desde el primer cilindro tomado hasta el último. En este estudio se analiza la suma de las puntuaciones obtenidas en la escala visual analógica para cada uno de los 10 cilindros tomados, encontrando diferencias significativas entre el grupo al que se le inyectó lidocaína al 1\% (media 16) y los controles (media 50) (13). Sin embargo nos parece importante objetivar que los factores que sí se han relacionado con la tolerabilidad de la prueba hayan sido la ansiedad con la que el paciente va a la prueba y el tono esfínteriano, ambas lógicas por otro lado, puesto que con una correcta premedicación usando alguna benzodiacepina podemos influir en ambos factores, calmando al paciente y aprovechando el perfil relajante muscular de este grupo farmacológico.

Son muchos los métodos anestésicos que se han ido empleando, desde la anestesia general, regional o sedación a la instilación de gel anestésico intrarrectal. Éste último ha sido una de los métodos más ampliamente utilizados gracias a su fácil aplicación. Se basa en la gran capacidad de absorción de la mucosa rectal, y se pretende una difusión del anestésico hasta alcanzar los nervios periprostáticos. En realidad la absorción rectal es a través de los plexos hemorroidales que conducen a la circulación sistémica y no a la zona periprostática. Hay algunos resultados favorables $(14,15)$ pero la mayoría de los autores no encuentra diferencias entre el empleo de lubricante con o sin lidocaína (16). Así ocurre en el trabajo de Desgrandchamps y colaboradores, en una muestra de 109 pacientes, en el que emplearon una escala cualitativa. Destacan sin embargo que el $90 \%$ dijo que el dolor que sufrieron fue nulo o leve. Tampoco hubo diferencias en las complicaciones (17). En nuestro trabajo hemos encontrado conclusiones similares, usando una EVA.

A raíz de los conocimientos anatómicos anteriormente descritos diferentes grupos han ido publicando pequeñas variaciones en la técnica de bloqueo nervioso periprostático, utilizando distintas zonas de punción. En general todos han encontrado diferencias clínicas y estadísticamente significativas en la comparación de dichas técnicas con placebo y lidocaína intrarectal (18), salvo el clásico trabajo de Wu y cols. en una cohorte únicamente de 40 pacien-

TABLA VI. COMPLICACIONES NO INMEDIATAS.

\begin{tabular}{|c|c|c|c|c|}
\hline & Grupo 1 & Grupo 2 & Grupo 3 & $p$ \\
\hline Hematuria: $61.194 \%(\%)$ & $23.881 \%$ & $13.433 \%$ & $23.881 \%$ & n.s. \\
\hline Días de hematuria & $\begin{array}{l}x=2.7 \\
\sigma=4.19 \\
R=0-15\end{array}$ & $\begin{array}{c}x=0.95 \\
\sigma=1.46 \\
R=0-5\end{array}$ & $\begin{array}{l}x=3.41 \\
\sigma=4.16 \\
R=0-14\end{array}$ & n.s. \\
\hline Rectorragia: $38.235 \%$ & $13.235 \%$ & $11.765 \%$ & $13.235 \%$ & n.s. \\
\hline Días de rectorragia & $\begin{array}{l}x=1.19 \\
\sigma=2.29 \\
R=0-10\end{array}$ & $\begin{array}{c}x=0.59 \\
\sigma=0.90 \\
R=0.3\end{array}$ & $\begin{array}{c}x=0.63 \\
\sigma=1.04 \\
R=0-3\end{array}$ & n.s. \\
\hline $\begin{array}{c}\text { Hemospermia: } 50.794 \% \\
\text { (No sabe: } 23.81 \% \text { ) }\end{array}$ & $15.873 \%$ & $14.286 \%$ & $20.635 \%$ & n.s. \\
\hline $\begin{array}{l}\text { Analgésicos en la tarde: } \\
\qquad 25 \%\end{array}$ & $10 \%$ & $5 \%$ & $10 \%$ & n.s. \\
\hline
\end{tabular}

$x=$ media; $\sigma=$ desv típica; $R=$ rango, sign=significativo, n.s. =no sign. 
tes, e inyectando sólo $5 \mathrm{~mL}$ bilateralmente, de manera lateral a las vesículas seminales (19).

Ozden y colaboradores comparan en su trabajo 7 grupos de 25 pacientes con distintas modalidades de bloqueo anestésico, incluyendo un grupo placebo. Combina la inyección de distintos volúmenes $(2.5,5$ o $10 \mathrm{cc}$ ) de lidocaína al $1 \%$ en la zona basal bilateralmente, con o sin inyección en el apex. Todos los grupos mostraron diferencias significativas con el placebo, excepto el grupo de $2.5 \mathrm{cc}$ inyectado en la zona basal bilateralmente. Los grupos en los que se inyectó $10 \mathrm{cc}$ de anestésico lograron un mejor control del dolor que en los que se inyectaron volúmenes menores. El grupo que mejor control del dolor logró fue el que utilizaba $10 \mathrm{cc}$ de lidocaína $1 \%$ en zona apical y basal (media de dolor 1.12), pero no se vieron diferencias estadísticamente significativas respecto al grupo en que sólo se aplicaba la anestesia en la zona basal (1.44), siendo necesaria la aplicación de más punciones. No encontraban diferencias en complicaciones (20).

En la tarde del procedimiento nuestros pacientes no muestran diferencias significativas respecto al dolor según el anestésico empleado, aunque fue mayor en el grupo 1, con una media nada despreciable de 4.06. Con idea de disminuir estas molestias Lee Elliott y cols. comparan el dolor en el momento de la biopsia, una hora después y en los siete días posteriores en 250 pacientes divididos en dos grupos, administrándosele $10 \mathrm{ml}$. de lidocaína $1 \%$ al grupo 1 , y $5 \mathrm{ml}$. de lidocaína $1 \%$ mas $5 \mathrm{ml}$. de bupivacaína $1 \%$ al grupo 2, con idea de aprovechar el distinto perfil de rapidez de actuación-duración del efecto de dichos anestésicos. No obtienen diferencias significativas en el dolor en el momento de la biopsia, pero sí pasada una hora. Lo que es más llamativo es que esta diferencia se mantiene estadísticamente significativa a lo largo de la primera semana, aunque con valores que clínicamente son poco significativos (en todo caso menores de 1 en la EVA) (21).

Otra aproximación a este problema es la de Ragavan y cols. En un estudio randomizado con tres brazos, comparan bloqueo neurovascular con lidocaína, lidocaína combinada con diclofenaco intrarrectal y esto último como única analgesia. Encuentran diferencias estadísticamente significativas respecto al dolor al tomar la biopsia entre grupos 1 y 2 con el $3 \quad(1.95$ vs 1.8 vs 3 respectivamente en escala 0-10). Lo más llamativo es que las diferencias también son significativas en la tarde (1.25 vs 0.3 vs $0.4)$, atribuyéndolas a una posible reacción inflamatoria debida a la lidocaína y atenuada por el diclofenaco. No encuentra diferencias ni a la hora ni al día siguiente, ni en complicaciones como hematuria, rectorragia, hemospermia o fiebre (22).
El de las complicaciones es otro de los aspectos que nos interesaba. A priori la inyección de un líquido (la lidocaína) atravesando la pared rectal debía ocasionar mayores complicaciones, sobre todo de tipo inflamatorio/infeccioso, que los otros métodos. Sin embargo, como hemos mencionado a lo largo de la discusión en la mayoría de trabajos no hay diferencias en las complicaciones encontradas según el tipo de anestesia empleada $(4,23,24)$, aunque Obek y cols. sugieren una mayor incidencia de fiebre y bacteriuria y menor de sangrado rectal en los pacientes en los que se emplea bloqueo neurovascular (25). En nuestra serie no hemos encontrado diferencias significativas ni inmediatas ni diferidas.

\section{CONCLUSIONES}

La ECO-TRB es una prueba que ocasiona molestias dolorosas no despreciables en la mayoría de pacientes, tanto de manera inmediata como diferida. El empleo de gel intrarrectal de lidocaína no ha demostrado en nuestra serie ser más eficaz que el uso de lubricante para el control de estas molestias. Por el contrario el empleo de lidocaína inyectada en ápex y vesículas seminales ha conseguido en nuestros pacientes un mejor control del dolor provocado por en el momento de la prueba, aunque no en horas posteriores, al menos de manera significativa estadísticamente. Se revela además como un método seguro, pues no provoca un mayor índice de complicaciones inmediatas o tardías.

El dolor provocado durante la prueba parece deberse fundamentalmente a la propia toma de biopsia, más que al transductor.

En nuestra muestra no hemos observado que factores como la edad o el volumen prostático se relacionen con mayor dolor durante la prueba. Sin embargo la ansiedad pre-prueba o el tono esfíteriano si han sido motivo de mayor sensación de disconfort. Posiblemente una premedicación correcta con benzodiacepinas pueda mejorar por tanto la tolerancia de la prueba, al incidir en ambos factores.

\section{BIBLIOGRAFÍA Y LECTURAS RECOMENDADAS (*lectura de interés $y^{* *}$ lectura fundamental)}

1. INSTITUTO NACIONAL DE ESTADÍSTICA. Nota de Prensa. http://www.ine.es/prensa/np444. pdf. 29 de enero de 2007.

2. NUPPONEN, N.; VISAKORPI, T.: "Molecular biology of progression of prostate cancer". Eur. Urol., 35: 351, 1999. 
3. GRUPO DE TRABAJO DEL ÁREA DE EPIDEMIOLOGÍA AMBIENTAL Y CÁNCER. CENTRO NACIONAL DE EPIDEMIOLOGÍA. INSTITUTO DE SALUD CARLOS III.: "La situación del cáncer en España 2005”. Disponible en: http://www.isciii.es/htdocs/pdf/cancer-msc. pdf. Citado 27 de abril de 2007.

4. HERRANZ, F.; DÍEZ, J.M.; CABELLO, R.: "Evolución de la técnica de biopsia transrectal ecodirigida de la próstata". Arch. Esp. Urol., 59: 385, 2006.

5. CONSEJERÍA DE SALUD. JUNTA DE ANDALUCÍA.: "Proceso asistencial integrado hiperplastia prostática benigna-cáncer". Disponible en http:// www.juntadeandalucia.es/salud/procesos/documentos.asp?idp=88. Citado 27 de abril de 2007.

6. NASH, P.A.; BRUCE, J.E.; INDUDHARA, R. y cols.: "Transrectal ultrasound guided prostatic nerve blockage eases systematic needle biopsy of the prostate". J. Urol., 155: 607, 1996.

7. SOLOWAY, M.S.; ÖBEK, C.: "Periprostatic local anestesia before ultrasound guided prostate biopsy". J. Urol., 163: 172, 2000.

8. SCHOSTAK, M.; CHRISTOPH, F.; MÜLLER, M. y cols.: "Optimizing local anesthesia during 10-core biopsy of the prostate". Urology, 60: 253, 2002.

*9. ÖBEK, C.; ÖZKAN, B.; TUNC, B. y cols.: "Comparison of 3 different methods of anestesia befote transrectal prostate biopsy: A prospective randomized trial". J. Urol., 172: 2004.

10. DJAVAN, B.; WALDERT, M.; ZLOTTA, A. y cols.: "Safety and morbidity of first and repeat transrectal ultrasound guided prostate needle biopsies: Results of a prospective European prostate cancer detection study". J. Urol., 166: 856, 2001.

11. RODRIGUEZ, L.V.; TERRIS, M.K.: "Risks and complications of transrectal ultrasound guided prostate needle biopsy: A prospective study and review of the literature". J. Urol., 160: 2115, 1998.

*12. FELTES, J.A.; PASSAS, J.; FELIP, N. y cols.: "La anestesia local mejora significativamente la tolerancia de la biopsia prostática". Arch. Esp. Urol., 59: 407, 2006.

13. KAVER, I.; MABJEESH, N.J.; MATZKIN, H.: "Randomized prospective study of periprostatic local anesthesia during transrectal ultrasound guided prostate biopsy". Urology, 59: 405, 2002.

14. ISSA, M.M.; BUX, S.; CHUNT, T. y cols.: "A randomized prospective trial of intrarectal lidocaine for pain control during transrectal prostate biopsy: The Emory University experience”. J. Urol., 164: 397, 2000.

15. RABER, M.; SCATTONI, V.; ROSCIGNO, M. y cols.: "Perinanal and intrarectal anaesthesia for transrectal biopsy of the prostate: A prospective randomized study for comparing lidocaine-prilocaine cream and placebo". BJU Int., 96: 1264, 2005.

16. GARCÍA MEDIERO, J.M.; MARTÍNEZ-PIÑEIRO LORENZO, L.; NÚÑEZ MORA, C. y cols.: "Uso de gel de lidocaína intrarectal en las biopsias transrectales ecodirigidas de próstata". Actas Urol. Esp., 27: 793, 2003.

*17. DESGRANDCHAMPS, F.; MERIA, P.; IRANI, J. y cols.: "The rectal administratinon of lidocaine gel and tolerance of transrectal ultrasonographyguided biopsy of the prostate : A prospective randomized placebo-controlled study". BJU Int., 83: 1007, 1999.

*18. RODRIGUEZ, A.; KYRIAKOU, G.; LERAY, E. y cols.: "Prospective study comparing two methods of anaesthesia for prostate biopsias: Apex periprostatic nerve block versus intrarectal lidocaine gel: review of the literature". Eur. Urol., 44: 195, 2003.

19. WU, C.L.; CARTER, H.B.; NAQIBUDDIN, M. y cols.: "Effect of local anesthetics on patient recovery after transrectal biopsy". Urology, 57: 925, 2001.

**20. OZDEN, E.; YAMAN, O.; GOGUS, C. y cols.: "The optimum doses of and injection locations for periprostatic nerve blockade for transrectal ultrasound guided biopsy of the prostate: a prospective, randomized, placebo controlled study". J. Urol., 170: 2319, 2003.

21. LEE-ELLIOTT, C.E.; DUNDAS, D.; PATEL, U.: "Randomized trial of lidocaine vs lidocaine/bupivacaine periprostatic injection on longitudinal pain scores after prostate biopsy". J. Urol., 171: 247, 2004.

22. RAGAVAN, N.; PHILIP, J.; BALASUBRAMANIAN, S.P. y cols.: "A randomized, controlled trial comparing lidocaine periprostatic nerve block, diclofenac suppository and both for transrectal ultrasound guided biopsy of prostate". J. Urol., 174: 510, 2005.

*23. RODRÍGUEZ -PATRÓN, R.; MAYAYO, T.; BURGOS, F.J. y cols.: "Tolerancia y complicaciones de la biopsia transrectal ecodirigida prostática ampliada a diez cilindros. Papel del bloqueo de haces neurovasculares con lidocaína". Arch. Esp. Urol., 58: 989, 2005.

24. AUTORINO, R.; DE SIO, M.; DI LORENZO, G. y cols.: "How to decrease pain during transrectal ultrasound guided prostate biopsy: A look at the literature". J. Urol., 174: 2091, 2005.

*25. OBEK, C.; ONAL, B.; OZKAN, B. y cols.: "Is periprostatic local anesthesia for transrectal ultrasound guided prostate biopsy associated with increased infectious or hemorrhagic complications? A prospective randomized trial". J. Urol., 168: $558,2002$. 